\title{
Classification of surface geometry behavior of cultural heritage surfaces based on monitoring change
}

Saha, Sunita, Siatou, Amalia, Sitnik, Robert

Sunita Saha, Amalia Siatou, Robert Sitnik, "Classification of surface geometry behavior of cultural heritage surfaces based on monitoring change," Proc.

SPIE 11784, Optics for Arts, Architecture, and Archaeology VIII, 117840H (8 July 2021); doi: 10.1117/12.2592387

SPIE. Event: SPIE Optical Metrology, 2021, Online Only 


\title{
Classification of Surface Geometry Behaviour of Cultural Heritage Surfaces based on Monitoring Change
}

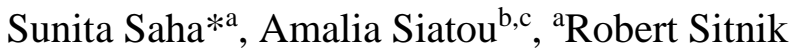 \\ ${ }^{\mathrm{a}}$ Faculty of Mechatronics Warsaw University of Technology, Poland; ${ }^{\mathrm{b}}$ Haute Ecole Arc \\ Conservation-Restauration, Neuchâtel, Switzerland ; 'Université de Bourgogne Franche-Comté, \\ Imagerie et Vision Artificiel, Dijon, France
}

\begin{abstract}
Efficient monitoring of large-scale cultural heritage monuments is of great interest in understanding alteration mechanisms that lead to substantial decision making for safeguarding them. This work presents a methodological approach for monitoring areas of the wall of King Jan III' palace Wilanów (Poland), where due to weathering the documentation of the surface changes became indispensable. Data from 3D scanning and registered data, representing different time intervals, were analysed to determine surface geometry changes. The goal was to develop a methodology which could detect each surface point based on grouping it with similar behaviour of local geometry changes. Further analyses, to determine the direction of change and the local geometry, were performed with the goal to extract information on the behaviour of changes and quantify them. By assigning a gradual scale, for the calculated displacement information regarding alterations can be visualized and measured. The methodology was based on calculating initially the general direction of change and then analysing the local geometry changes based on considering neighbouring points obtained from the spherical search kernel of each surface point. The neighbouring points were then compared to weathered dataset point cloud, by calculating the Euclidean distance and data segmentation, based on histograms of local distance analysis, was produced. Each histogram was fitted to the kernel distribution curve and bandwidth parameters, with similar points, were identified and segmented for changes corresponding to different time intervals. Initial evaluation on the case study shows the ability of the proposed methodology to detect even minor surface displacements.
\end{abstract}

Keywords: 3D scanning, Geometry change, Local geometry, Segmentation, Monitoring, Cultural heritage, Classification.

\section{INTRODUCTION}

The significance of recording and monitoring Cultural Heritage $(\mathrm{CH})$ monuments and sites has been well established as one of the most important steps for their assessment, ensuring sustainability and long-term preservation [1-3]. This paper focuses mainly on monitoring, which refers to the collection of data pertaining to an object and its environment over time [3]. This is a common practice in the field of $\mathrm{CH}$ documentation, that helps in understanding the object or monument and correlate its state of preservation (condition) to the surrounding environment, while tracking and characterizing any possible change on its surface. Some of the most important features in monitoring is visualisation and quantification of surface alterations. Visualization usually involves photographs, sketches, cartographies, 3D modelling, 3D texture mapping etc. of the examined surface over time [4] whereas quantification is still an open issue since evaluation of change is usually done empirically by field experts (i.e., conservator-restorers, architects) and through visual comparisons at different time intervals. Even though experts' assessment is invaluable, minor changes cannot easily be detected.

In parallel, advancements in $\mathrm{CH}$ digitization can provide automations and quantifications of surface observations with high accuracy and repeatability. One of these techniques involves 3D scanning since it can capture the surface texture and create accurate and realistic representation of $\mathrm{CH}$ surfaces. Moreover, scans at different time intervals can, through appropriate registration, provide a continuous dataset that can in return provide quantifiable information of the surface and thus determine the presence or absence of changes. This technology is not related to alteration mechanisms and therefore can be used as an unsupervised methodology for examining even complex changes on $\mathrm{CH}$ surfaces.

This work proposes a change-based segmentation method, for application in the field of $\mathrm{CH}$, to perform monitoring in correlation to observed alterations provoked by weathering conditions. The novelty of the proposed work is the 3D analysis of geometrical changes on $\mathrm{CH}$ surfaces as well as the identification and naming of the behaviour of the change occurrence

Optics for Arts, Architecture, and Archaeology VIII, edited by Haida Liang, Roger Groves, Proc. of SPIE

Vol. $11784,117840 \mathrm{H} \cdot$ @ 2021 SPIE · CCC code: $0277-786 \mathrm{X} / 21 / \$ 21 \cdot$ doi: $10.1117 / 12.2592387$ 
for each surface point. This finds ground in the field of conservation-restoration for the documentation and quantification of change that can lead in decision making for the prevention of further damage by using 3D digital representation.

The proposed methodology was evaluated for a case study at the Museum of King Jan III's Palace at Wilanów (Warsaw, Poland). Parts of the building's interior and exterior as well as selected collections have also been examined through different techniques and methods to evaluate the documentation, management, and sustainability of this cultural heritage monument $[5,6]$. In this project, the aim is to identify surface changes based on 3D scans acquired at different time intervals and quantify them through the change-based segmentation method. Parts of the façade that had visible signs of surface degradation were selected for this purpose.

\section{RELATED STUDIES}

In the field of $\mathrm{CH}$ there is limited number of references on imaging and change measurement of the surface geometry using the segmentation approach. Regarding the state of the art, no related work has been found in terms of assessment of surface geometry changes overtime. Previous works, dedicated to $\mathrm{CH}$ change detection, were mostly focusing on global geometry change assessment and segmentation for shape retrieval for single phase data (time instances). Meanwhile, various researchers are developing methods for geometric segmentation in different fields like medical imaging, remote sensing, etc. Understanding 3D data is a core issue in 3D representation and is fundamental to applications such as robotics, autonomous driving, augmented reality and construction industry. Among various 3D representation problems, geometric segmentation allows accurate object boundaries retrieval, as well as assigning labels to objects in 3D space [7]. In this section the analytical approaches developed and dedicated to $\mathrm{CH}$ change detection by geometry comparison are presented.

In [5], the change assessment of global geometry was evaluated considering the distance calculation for each point of the reference dataset, to the calculated surface mesh of the weathered dataset. In [8], change assessment was performed by distance calculation between a point on the reference dataset to the immediate nearest point to the weathered dataset. Their work was limited to distance calculations of $\mathrm{C} 2 \mathrm{M}$ (Cloud to Mesh) and $\mathrm{C} 2 \mathrm{C}$ (Cloud to Cloud) that according to [4] through an evaluation of various ways of geometry comparison methods $\mathrm{C} 2 \mathrm{C}$ and $\mathrm{C} 2 \mathrm{M}$ distance calculation were proven to be unable to quantify the amount of change on the surface. Though both papers were dedicated to $\mathrm{CH}$, the developed methodology in [9], filled the three major key characteristics where the distance calculation was performed directly on point clouds without meshing, computes the local distance between two-point clouds along the normal surface direction and it estimates the confidence level for each computation depending on the point cloud roughness and registration error. Additionally, this research was not considering the complexity of a $\mathrm{CH}$ object's surface and it was focusing on the point cloud comparison in various application fields and for general purposes. In [10], geometry comparison was performed using M3C2 (Multiscale Model to Model Cloud Comparison) distance calculation, between the reference dataset and the weathered dataset, and claimed that this methodology is efficiently assessing global change for monitoring $\mathrm{CH}$ artefacts but adopting the calculation would be expensive. In [11], cross-time registration was introduced among other techniques for the documentation and evaluation of cleaning tests, conducted on various objects, in an underwater environment. For the implementation physical markers were used with the goal to compare areas before and after cleaning by identifying corresponding points in the surrounding of the cleaned area. The known distance measurement value was rescaled for the distance calculation on the entire surface to avoid registration. In [12], an analysis was presented, of a change detection process of a multitemporal data set which was based on the interpretation of depth map images. Several maps were generated by orthophotos of a mock-up object that was artificially aged to acquire a multitemporal data set. To have accurate size of input from both the datasets, the set-up needed resampling and resizing of the input data for each computation. Additionally, the experimental data were referring to artificial conditions, so the change detection is not fully representing real weathering conditions and their complexity. In [13], a method was presented to evaluate the potential of an easy-to-use and intuitive tool to produce degradation maps, and the identification of areas which have been affected by chemical and physical changes over time. The Multivariate Alteration Detection (MAD) algorithm was selected over Principal Component Analysis (PCA). The main principle of MAD is to make the images as similar (i.e., correlated) as possible, before computing their difference to find two sets of linear combinations of the original variables. For the 3D change detection, different distance thresholds were identified, corresponding to different changes at varying scales done in Cloud Compare. However, to fit the data from different time intervals a common coordinate system, for both surveys, was selected to identify natural features on the surface of the object. Furthermore, it is implicit that the input images from different times should have the same size and occupy the same physical space which in the examined cases it required 
cropping the images. However, assessing global geometry change is not enough to understand the change behaviour of local geometry of each surface point.

In [14], a point cloud segmentation and classification using simple geometrical rules, such as convex hull areas and circularity values was presented. They proposed an automated approach of segmentation, identification, and classification of point clouds into architectural elements addressing the detection of attics and structural supports. The work was promising in minimizing as much as possible human intervention during the process of segmentation, but difficulties arose when applying the algorithm by default, as the function arbitrarily takes the middle altitude cross-section of the point cloud to perform the detection part.

Studies in industrial applications for the geometric segmentation based on octree-based region growing algorithms are also presented [15, 16]. In [17], a novel method of point clouds segmentation is proposed based on Euclidean clustering and multi-plane extraction to cope with overhanging objects, such as tree branches, with the assistance of a hybrid elevation map. They claimed accuracy and efficiency of their results over the existing methods. But their goal was to develop segmentation on one phase geometry data and was not dedicated to change assessment. The work [18], presented a method to segment geometric features in discrete geometric models based on region growing algorithms assessing the similarity of point type possibility. But the method proposed requires the differential geometric properties (normal and principal curvatures) that must be estimated beforehand at each node of the triangular mesh which was unable to identify the smooth transition zones.

\section{CHANGE ASSESSMENT}

In this paper, the assessment of changes on the $\mathrm{CH}$ surfaces is performed in two sections of global and local geometry comparison. The global geometry change aims in calculating the Euclidean distance between a considered point from a reference dataset $(\mathrm{T})$ to its corresponding change point on the weathered dataset $(\& \mathrm{~T})$. The local geometry change is analysed considering points on the reference dataset and its nearest neighbour points distance behaviour to the corresponding changed points for each locality. Segmentation is then proposed based on kernel distributions of the local geometry histograms. The respective flowcharts are presented in Figure 1 and 2 for the global and local geometry change analysis. The advantage of the proposed methodology is that change assessment does not depend on the size of the $\mathrm{CH}$ objects or its composition.

\subsection{Global geometry changes assessment}

For each considered point of the $\mathrm{T}$ the normal vector of the point and the displacement vector of the exact corresponding change point from the \& $\mathrm{T}$, is computed. However, there are several factors like roughness of the surface, noise on the data, direction of change etc. that might affect the selection of corresponding change-point from the weathered dataset which were described in [9]. In this work, the corresponding changed point is explored within a specified volume along the line crossing the considered point and its normal vector. The nearest point is therefore computed along the introduced line and the projected line. Then, the signed distance is computed to evaluate the negative or positive change in comparison to the reference surface. In case no changed point is detected within the specified volume the distance is set as NULL. However, a user can adjust the search volume to find the changed point which basically corresponds to a nearest point and not an actual changed point. An example of this scenario where a point on $\mathrm{T}$ could not find a corresponding changed point from $\& \mathrm{~T}$, due to the detachment of a part of the surface between monitoring periods, is shown in (4.3). The calculation is more accurate if the corresponding changed points can be found within a small search volume, adequate to the dataset examined, which requires denser input data and acquisition in cross time registration. 


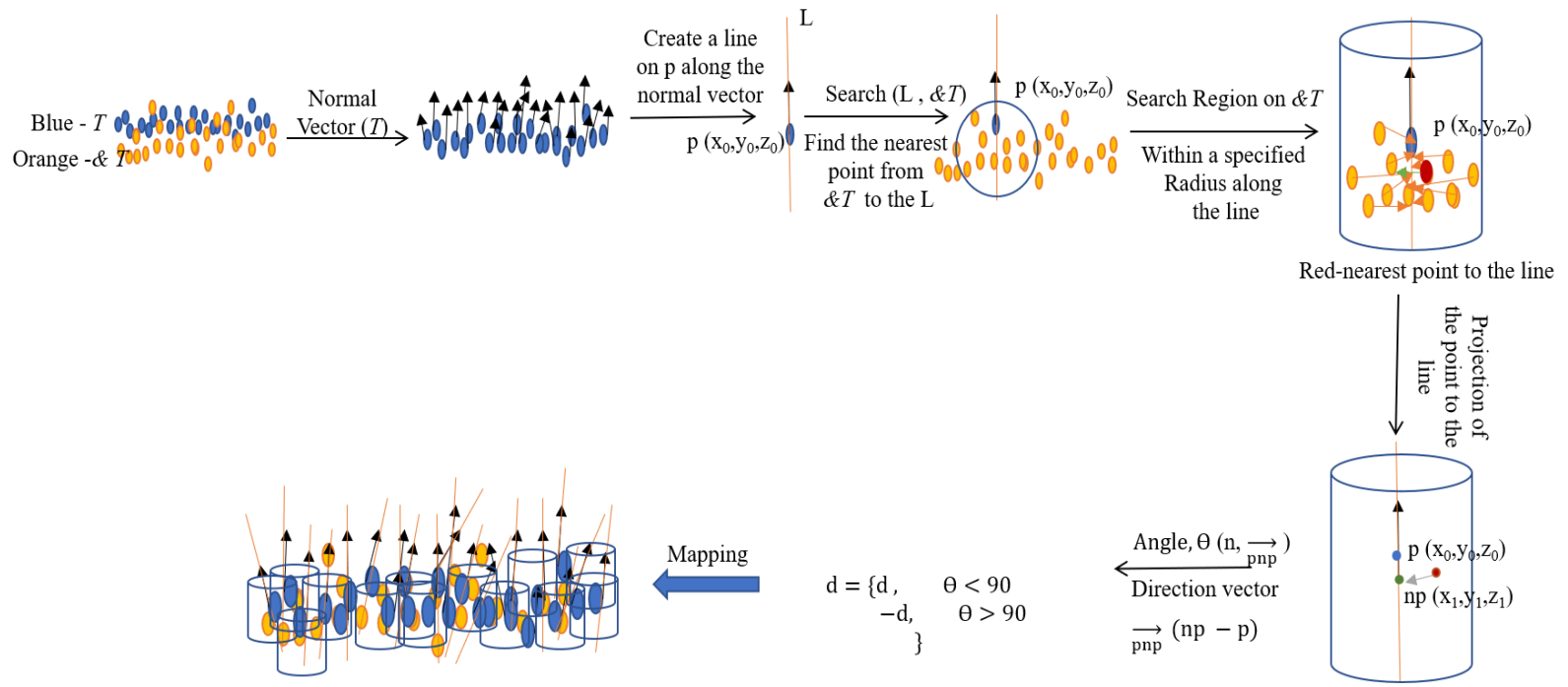

Figure 1 Global geometry change detection flowchart.

This global geometry assessment can be used to estimate the overall change and locate displacements corresponding to surface alterations. The chance of having a bias is related mainly to the quantification of the geometry change due the cross-time registration. Moreover, the global geometry change assessment itself is not accurate enough to adequately present or quantify the changes on a monument's surface during monitoring. More specifically, the global geometry change analysis alone cannot identify the similar local geometry change behaviour of each points on the surface. In this work the focus is on assessing and classifying the surface geometry change behaviour of each surface point and its neighbouring points.

\subsection{Local geometry changes assessment}

To assess the local geometry, namely the change for each surface point from $\mathrm{T}$, the analysis considered the neighbouring points within a specified volume. After finding the neighbouring points from $\mathrm{T}$, the exact k-neighbourhood points were examined in comparison to \&T to create local distribution for each surface point. The search volume was normalized to the calculated sampling distance of the input data to make the calculation independent to the resolution of the data. A scaling factor was considered, where an end user can scale the volume based on user experience and density of the input data.

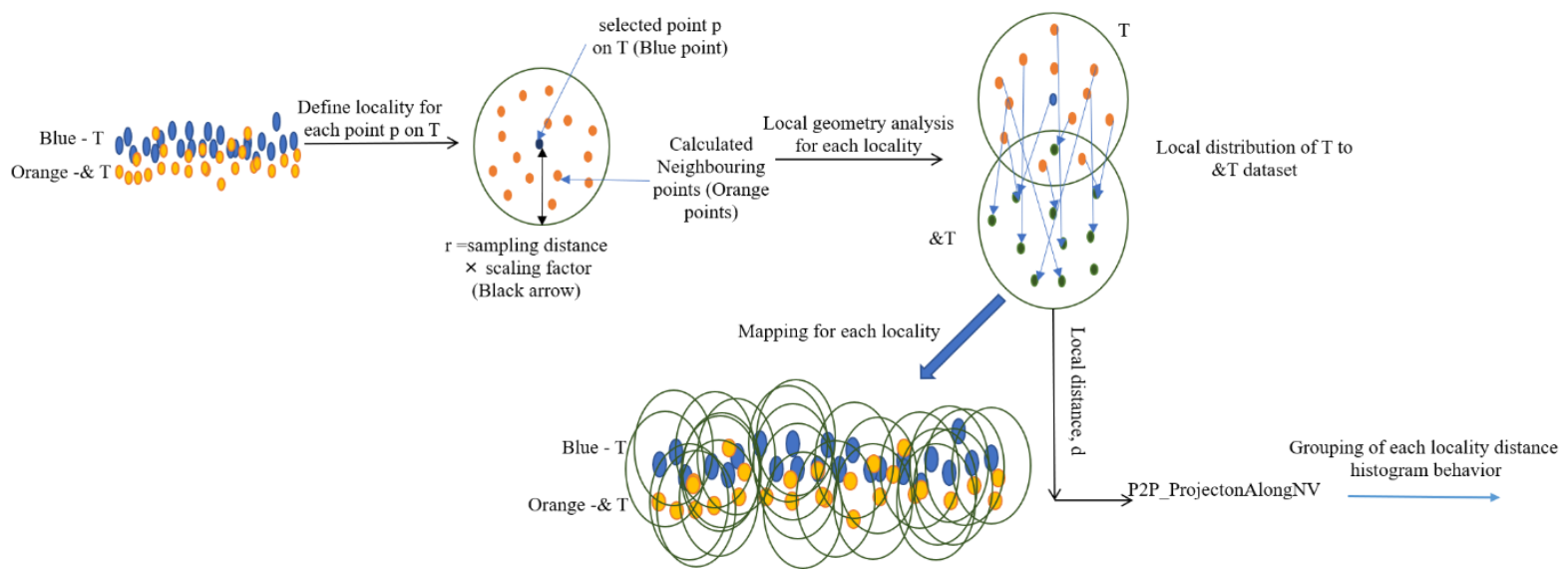

Figure 2. Local geometry analysis flowchart 
For each local distribution (fig.2), the distance was calculated following the global geometry analysis. To achieve this local distribution and the calculated local distance histograms are considered to identify the behaviour of each surface point and classify them to a given change category. The stored local distance histogram for each surface point from $\mathrm{T}$ is then passed to the segmentation method described below.

\subsection{Segmentation}

Segmentation was performed considering several calculated parameters from the local distance histogram, factors from the input data and the user's input threshold values. The calculated local distance histogram was fit to a kernel distribution curve resulting in the extraction of features for performing inspection on their behaviour [19, 20]. The mean and bandwidth of the histogram was calculated from the generated local distance histogram for each point. The kernel density estimating, $f_{n}$, of a set of $n$ points from a density $f$ is defined as:

$$
f_{n}(x)=\sum n_{j}=1 K\left\{\left(x-X_{j}\right) h\right\} n h
$$

where $\mathrm{K}$ is the kernel function, and $\mathrm{h}$ is the smoothing parameter or window width.

The optimal width for a bandwidth calculation of a histogram, according to Silverman's DATA PLOT, is:

$$
1.06 \times \mathrm{s} \times \mathrm{knn}-\frac{1}{5}
$$

where knn is the number (No) of points in the raw data (No of points inside each locality) and s is the sample standard deviation of the raw data.

From the input data, data sampling distance (sd) was calculated, and data noise is parameterized to make the method insensitive to the external noise. The noise was calculated considering RMS (root mean square) distance for randomly chosen points on the surface to the fitted plane from the surface points. Also, for each local distribution plane fitting errors, $\mathrm{e}_{1}$ and $\mathrm{e}_{2}$, are calculated from both $\mathrm{T}$ and \& $\mathrm{T}$, respectively.

The calculated mean $(\mathrm{m})$, bandwidth $(\mathrm{b})$ and noise $(\mathrm{N})$ are normalized with the sampling distance, $\mathrm{such}$ as $\mathrm{m}=\mathrm{m} / \mathrm{sd}, \mathrm{b}=$ $\mathrm{b} / \mathrm{sd}$ and $\mathrm{N}=\mathrm{N} / \mathrm{sd}$. Several user inputs are considered as input to the segmentation method to define major and minor values in either case of positive or negative change on the surface. Two threshold values namely positive threshold (pT) and negative threshold (nT), were assigned. These thresholds will help to quantify the major and minor changes on the surface geometry and their selection is based exclusively on field experts, the overall size of the object and the expected change. These values can be chosen more precisely based on the calculated minimum and maximum values in the global geometry. Another threshold value, named registration threshold (rT), is incorporated, as shown in Eq3, to avoid the crosstime registration error and to bring more accuracy to the segmentation method. The default value of this threshold is kept at 1 , but it can be user defined. This threshold value is a ratio of normalized noise and No. of points inside each locality (N/knn). However, to properly set this threshold a known reference point of an unchanged part of the surface must be calculated. An exemplary comparison is shown in Table 8 in the results section, where a 16 days' time interval is considered that would not result in changes on the examined surface. The conditions are set thereafter, to group the similar behaviour surface points and categorize them to a certain type of changes. The conditions set and identified changes are shown in the following equations of $3-8$.

$$
\begin{gathered}
\text { Change Type I }=\mathrm{b}<\left(\frac{\mathrm{N}}{\mathrm{knn}}\right) \times \mathrm{rT} \& \&|\mathrm{~m}|<\left(\frac{\mathrm{sd}}{\mathrm{knn}}\right) \times \mathrm{rT} \\
\text { Change Type } \mathrm{II}=\mathrm{m}>0 \& \& \mathrm{~m} \leq \mathrm{pT}
\end{gathered}
$$


1. $\mathrm{b}<\mathrm{N}$

2. $((\mathrm{e} 1, \mathrm{e} 2)<\mathrm{N})$

3. $((\mathrm{e} 1, \mathrm{e} 2)>\mathrm{N})$

Chnage Type III $=\mathrm{m}<0 \& \& \mathrm{~m} \geq \mathrm{nT}$

1. $\mathrm{b}<\mathrm{N}$

2. $((\mathrm{e} 1, \mathrm{e} 2)<\mathrm{N})$
3. $((\mathrm{e} 1, \mathrm{e} 2)>\mathrm{N})$

Change Type IV $=m>0 \& \& m>p T$

1. $\mathrm{b}<\mathrm{N}$

2. $((\mathrm{e} 1, \mathrm{e} 2)<\mathrm{N})$

3. $((\mathrm{e} 1, \mathrm{e} 2)>\mathrm{N})$

Chnage Type $\mathrm{V}=\mathrm{m}<0$ \&\& $\mathrm{m}<\mathrm{nT}$

1. $\mathrm{b}<\mathrm{N}$

2. $((\mathrm{e} 1, \mathrm{e} 2)<\mathrm{N})$

3. $((\mathrm{e} 1, \mathrm{e} 2)>\mathrm{N})$

ChangeType VI = Unknown

\subsection{Visualization}

Colormap visualization of the calculated distances in the global geometry analysis is presented based on calculated minimum and maximum distance obtained from each individual geometry comparison. This will help the user to set and correlate the threshold values of positive or negative change needed for the segmentation and quantify it accordingly.

For the visualization of the segmentation method and the identified changes, a colour scale is assigned to each category. To make it user-friendly the category of Change Type I is considered as 0 which basically represents No- Change (Blue). The Change Type II and Change Type IV are sets of triple segments defined as +1 and +2 corresponding to minor (Light blue/cyan/light cyan) and major (Light green/green/dark green) positive change, respectively. Similarly, for the Change Type III and Change Type V that are set as -1 and -2 for minor (Light yellow/yellow/orange) and major (Light red/ red/ dark red) negative change, respectively. The unknown (brown) category of change is presented as " $\times$ " in the visualization.

Table 1. Classification of change based on segmentation

\begin{tabular}{|l|l|l|l|}
\hline Type of Change & Scale & Colour & definition \\
\hline Change Type I & 0 & blue & No Change \\
\hline Change Type II & +1 & Light blue/cyan/light cyan & minor positive change \\
\hline Change Type III & -1 & Light green/green/dark green & minor negative change \\
\hline Change Type IV & +2 & Light blue/cyan/light cyan & major positive change \\
\hline Change Type V & -2 & Light green/green/dark green & major negative change \\
\hline Change Type VI & $\mathrm{X}$ & brown & unknown change \\
\hline
\end{tabular}

It should be noted that the colormap representing the global geometry analysis is not related to the visualization of the segmentation method. 


\section{RESULTS AND DISCUSSION}

This section presents the results obtained from the application of the aforementioned methodology on parts of the façade of the Museum of King Jan III's Palace at Wilanów (Warsaw, Poland).

\subsection{The case study}

The proposed methodology was applied to selected areas of the façade of the Museum of King Jan III's Palace at Wilanów (Warsaw, Poland), a baroque roller residence dating back to 1677 [21]. The necessity to quantify changes on the surface as part of its condition assessment has led to a project aiming in the automation of acquisitions and data processing at different time intervals [5]. The same data are examined with the proposed methodology.

The initial evaluation was based on structured light scanning and data processing in global and local scale for a quantitative assessment of the change in surface geometry. To assist registration markers were fixed around the area of interest. The results were evaluated by experts and by calculating the surface geometry of the histograms [5].

Areas of interest, with a size of $120 \times 120 \mathrm{~mm}$, from the facade of the building were selected by experts based on potential damage risk and ease of access [5]. In the current paper, two of the above-mentioned areas, showing initial signs of damage (i.e., surface cracks, material loss) were selected to be further assessed with the proposed methodology.

These correspond to area No6, on which a small detachment occurred during the monitoring period, and area No10 that showed visible cracks on the surface.

\subsection{Methodology}

To evaluate change over time, acquisition from different time intervals were evaluated. Due to weather conditions the selected time intervals were not regular. Surface data were acquired as shown in Table 2. For the purposes of assessing possible changes all possible combinations were considered, namely distance between different time intervals and distance in comparison to the reference (Session 1).

Table 2. Monitoring periods

\begin{tabular}{|l|l|l|}
\hline Time Interval & Date & Days \\
\hline Session 1 & $30-31 / 05 / 2018$ & 0 \\
\hline Session 2 & $20-21 / 08 / 2018$ & 81 \\
\hline Session 3 & $03-05 / 09 / 2018$ & 97 \\
\hline Session 4 & $11-12 / 10 / 2018$ & 134 \\
\hline
\end{tabular}

The methodology applied considers the global and local distance, as described in 3.1 and 3.3, as well as the segmentation based on default or user generated thresholds. For the case study under examination, these were applied to selected areas of interest but also locally on Regions of Interest (ROI) from the same areas that presented features that were related to further damage.

Measured distances vary according to input data and are system defined for the global distance but can be selected for the segmentation process. Regarding segmentation the default value $\mathrm{rT}=1$ was selected. However, it was decided to evaluate a user-defined threshold, based on the assumption that a time interval of 16 days (S2-S3) is too short to have quantifiable changes on a wall surface, unless major events are recorded. Therefore, an rT=200 was defined as a search radius value. 


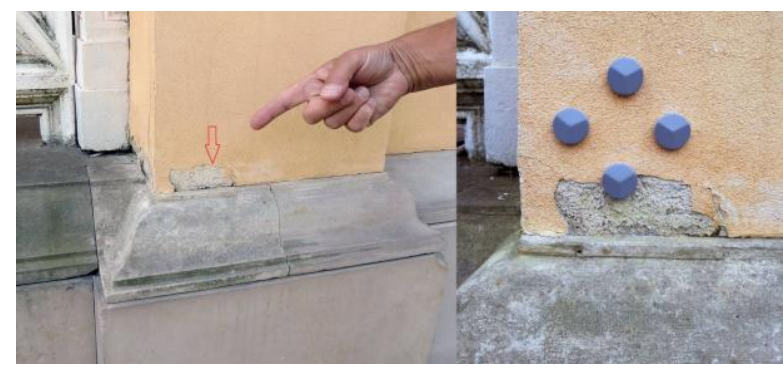

Figure3. Measurement fields: left - one of 12 measurement fields indicated as significant for monitoring changes in time, right same field equipped with reference markers.

\subsection{Results}

\section{Area No6}

In area N06 a visual change was detected between S1 and S2 since a small fragment was detached (approximately 20x5 $\mathrm{mm}$ ) from the surface (Table 3). In the visualizations below, the surface detachment is presented as NULL (see section 3.1), since it corresponds to total loss from the surface. To investigate the surface behaviour, the area was selected as ROI1 and local geometry analysis was performed (ROI1-Table 9).

Global distance measurements vary for each time combination. The values between time intervals show a variation between minor positive and negative displacements with higher fluctuations between S1-S2 and S2-S3, on the contrary the time intervals S2-S4 and S3-S4 show minor negative change (Table 4). The total scale of displacement is between 1 or $2 \mathrm{~mm}$ and therefore alignment errors cannot be excluded.

In parallel, the global distance calculations, from the reference, measures a more constant displacement of a scale of $2 \mathrm{~mm}$. The general trend shows a higher degree of change within the first 97 days of observations and a less significant displacement at 134 days (Table 5). The general trend of the surface corresponds to areas with negative and positive values. In terms of monitoring the condition of the surface there is evidence that a small part is moving towards positive values, this part corresponds to an area with observed cracks and therefore could be assigned to evidence of a risk for detachment. This area has been selected as ROI-2 and its local geometry was analysed (Table 10). Additionally, the upper right and lower left parts also show a movement to the positive direction but of a much smaller magnitude.

Segmentations between time intervals (Table 6) and in comparison, to reference (Table 7) with a default threshold agrees with the global distance measurements respectively (Table 4, 5), but interpretation of the results is not straight-forward.

Contrarily, segmentation based on expert-defined threshold presents a different visualisation, but with the same results in principle, depicting the same risk area (ROI02) as described above and a general positive displacement on the upper right and lower left regions (Table 8).

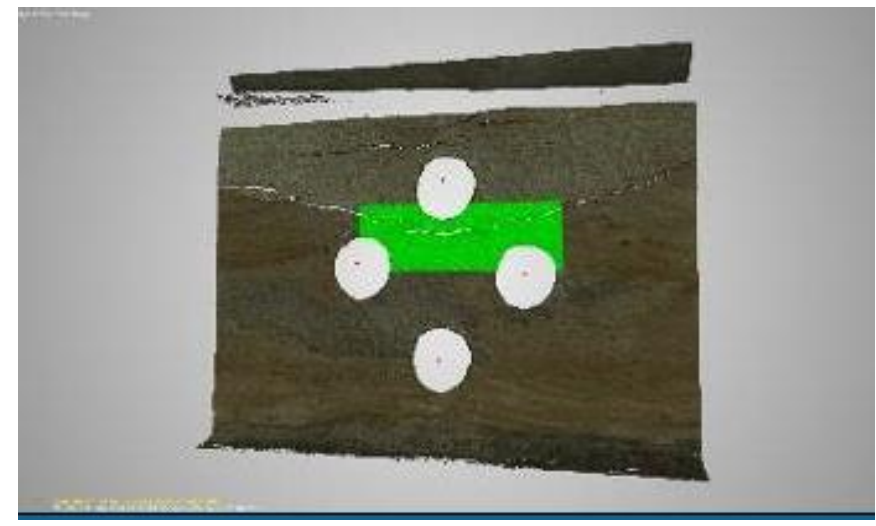

Figure 4. Area No. 6, $(70 \times 22 \mathrm{~mm})$ 
Table 3. Area No6- 3D mesh of the different time intervals

\begin{tabular}{|c|c|c|c|}
\hline Session1 (S1) & Session 2 (S2) & Session 3 (S3) & Session 4 (S4) \\
\hline $2018-05-30 / 31$ & $2018-08-20 / 21$ & $2018-09-03 / 05$ & $2018-10-11 / 12$ \\
\hline & & & \\
& & & \\
\hline
\end{tabular}

Table 4. Global Distance calculation between time intervals (default registration threshold)

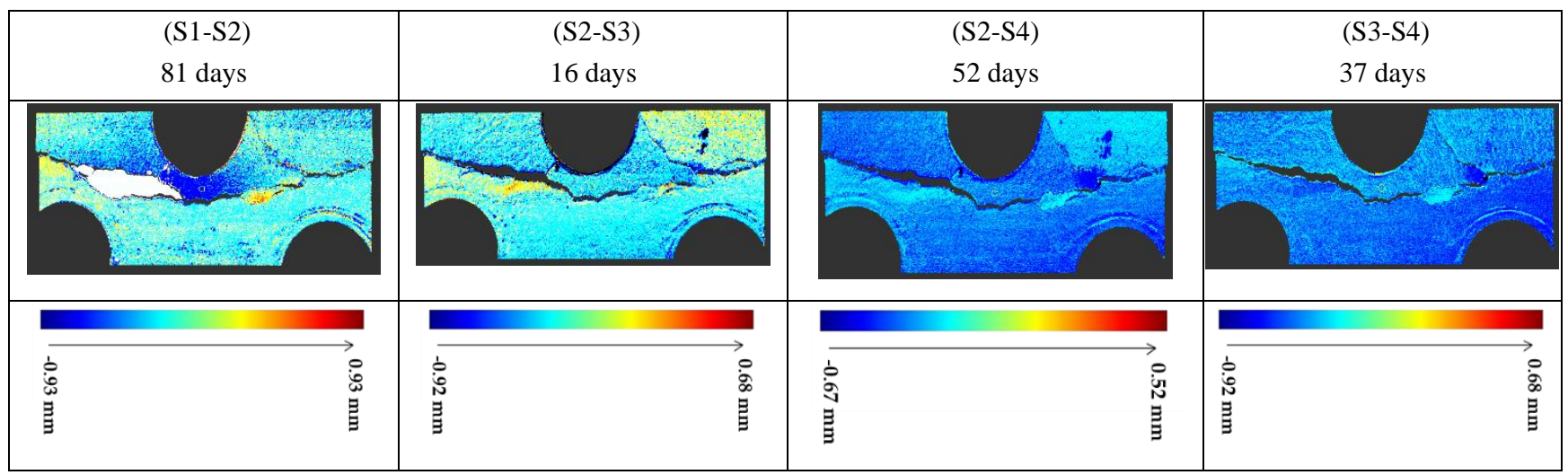

Table 5. Global Distance calculation from reference (default registration threshold)

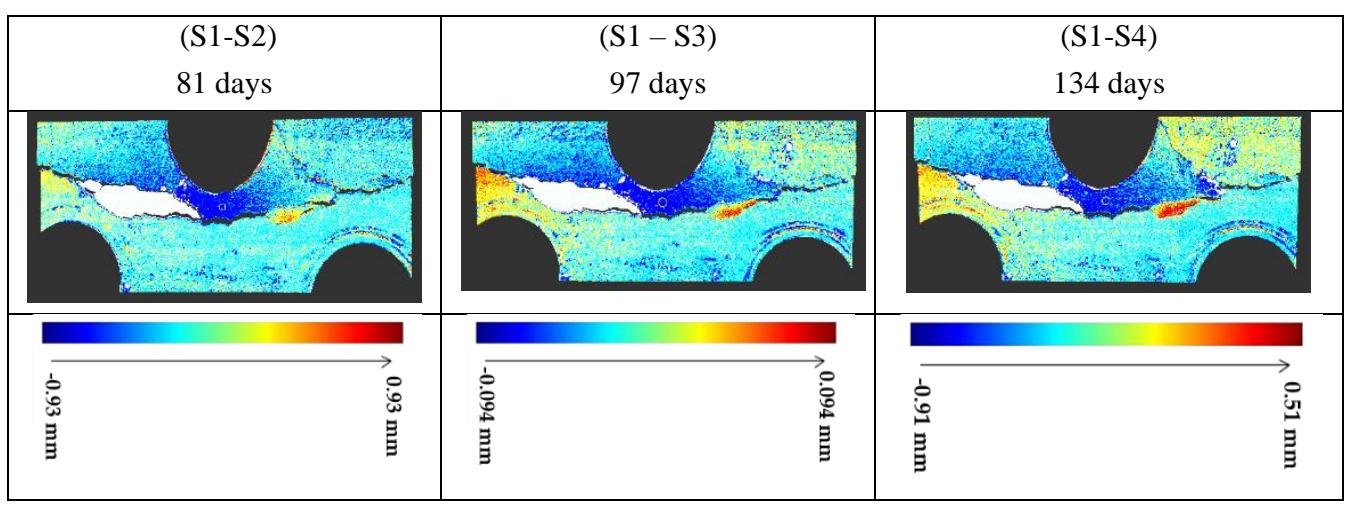

Table 6. Segmentation between time intervals $(\mathrm{pT}=0.5 \mathrm{~mm}, \mathrm{nT}=0.5 \mathrm{~mm}, \mathrm{rT}=1)$

\begin{tabular}{|c|c|c|c|}
\hline $\begin{array}{c}\text { (S1-S2) } \\
81 \text { days }\end{array}$ & $\begin{array}{l}(\mathrm{S} 2-\mathrm{S} 3) \\
16 \text { days }\end{array}$ & $\begin{array}{c}\text { (S2-S4) } \\
52 \text { days }\end{array}$ \\
\hline & & & \\
\hline
\end{tabular}




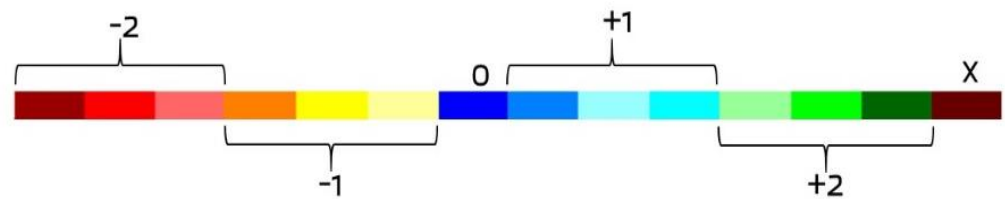

Table 7. Segmentation from reference value with default threshold $(\mathrm{pT}=0.5 \mathrm{~mm}, \mathrm{nT}=0.5 \mathrm{~mm}, \mathrm{rT}=1)$

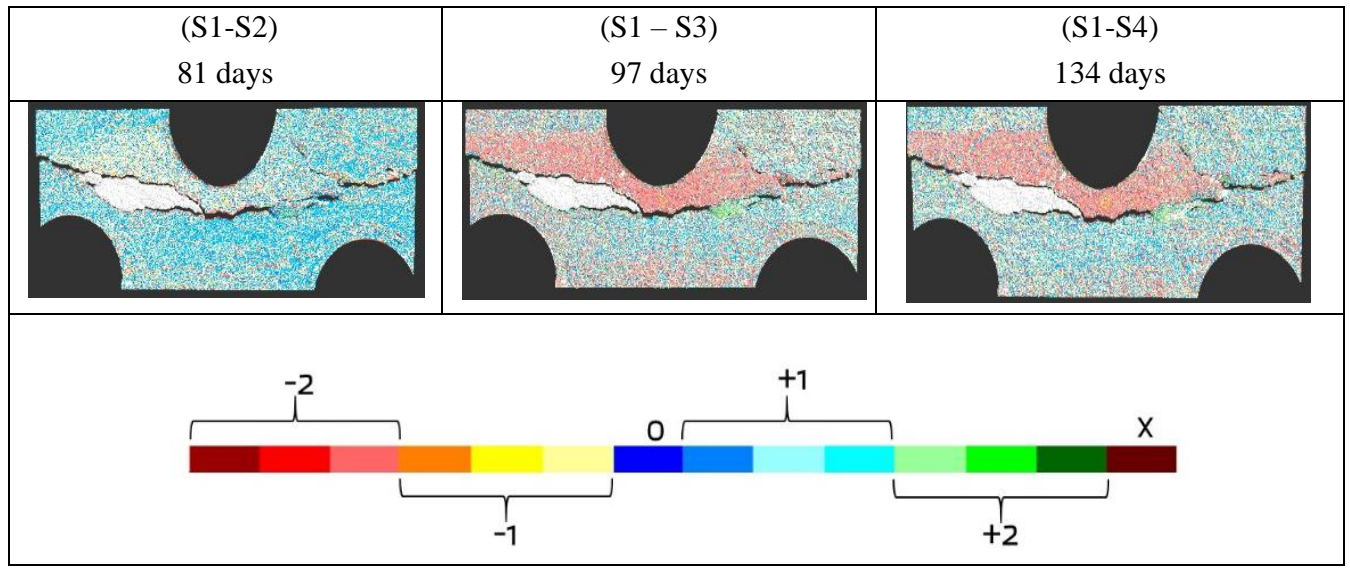

Table 8. Segmentation from reference value with user defined threshold $(\mathrm{pT}=0.5 \mathrm{~mm}, \mathrm{nT}=0.5 \mathrm{~mm}, \mathrm{rT}=200)$

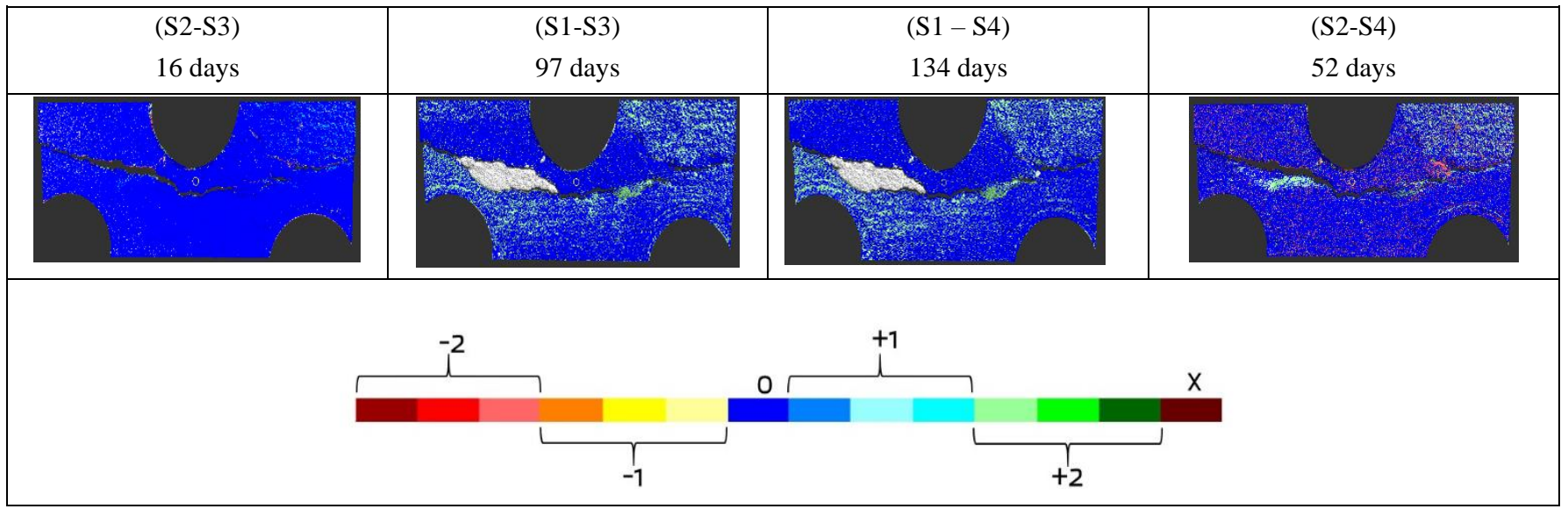

\section{ROI 1}

For the examination of local geometry of ROI 1, namely the area with the visible detachment, the respective height maps were also inspected for the S1 and S4. The search radius for the global distance calculation was increased to include the detached area.

It is evident that height maps are difficult to interpret especially since the scale is defined by the point cloud in each session and therefore quantifying and visualizing changes can lead to erroneous results.

Global distance measurements correspond to an area of change of about $5 \mathrm{~mm}$ denoting the detached fragment. A trend of displacement on the surface is visible depicting areas with negative and positive values, with a concentration of higher positive values on the centre of the detachment between S1-S4. The same trend becomes more evident in the case of 
segmentation where the area of the detachment is divided into two segments one presenting positive values and one negative. These segments represent the measured changes of the different time session in comparison to the reference (S1). Moreover, as noted in the examination of the entire area No6, by changing the rT threshold the visualisation of the change trend becomes more easily identifiable.

Table 9. Results on ROI-1 chosen from Area No 6 with default registration threshold and chosen registration threshold

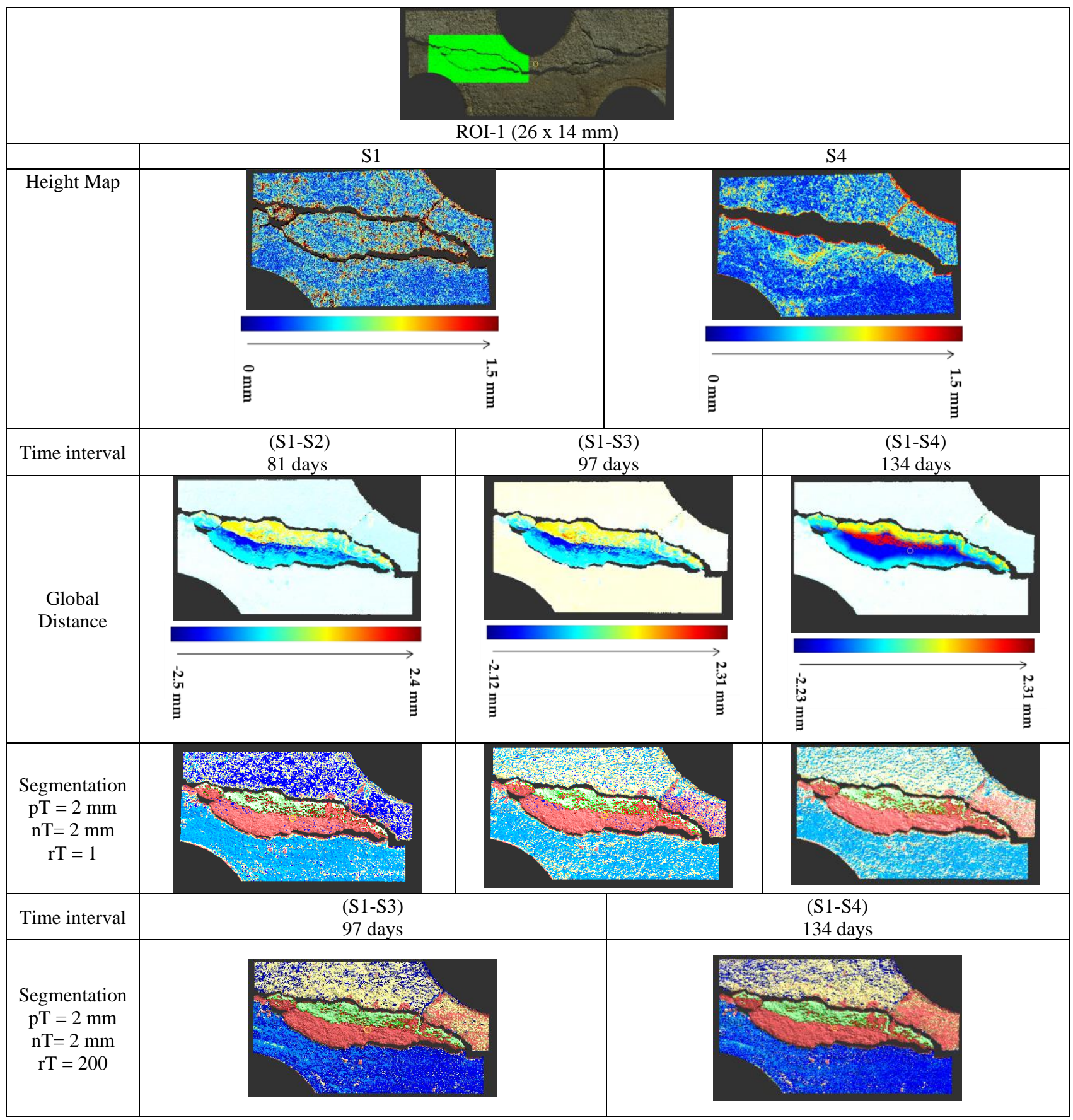




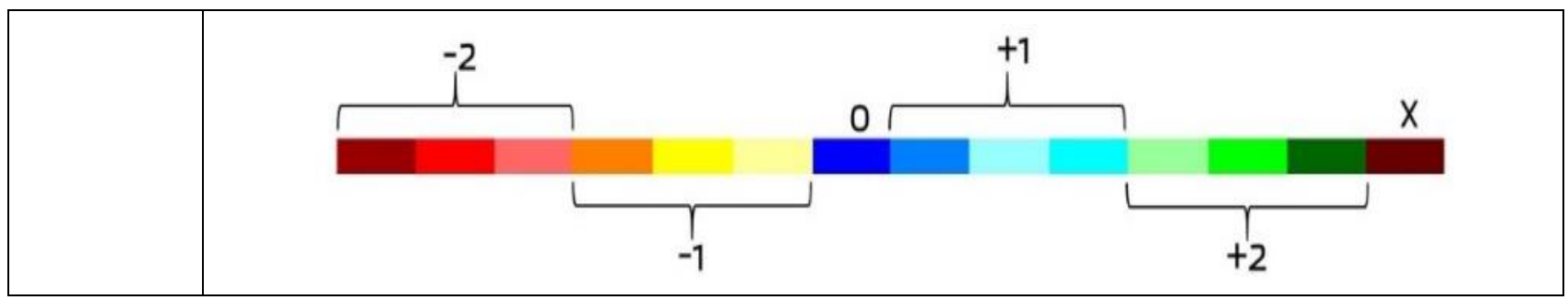

\section{ROI-2}

Similarly, to ROI-1 the height maps are difficult to interpret, and they mainly represent maximum values corresponding to the edges of the cracks. The global distance measurements for the selected regions shows a positive change over time for the part that is situated between the surface cracks enhancing the assumptions of an area of risk for detachment, even though the measured distance is around $0.22 \mathrm{~mm}$ to the reference. This is also visualized in the segmentation where this information becomes clearer with the expert-defined threshold.

Table 10. Results on ROI-2 chosen from Area No 6 with default registration threshold and chosen registration threshold

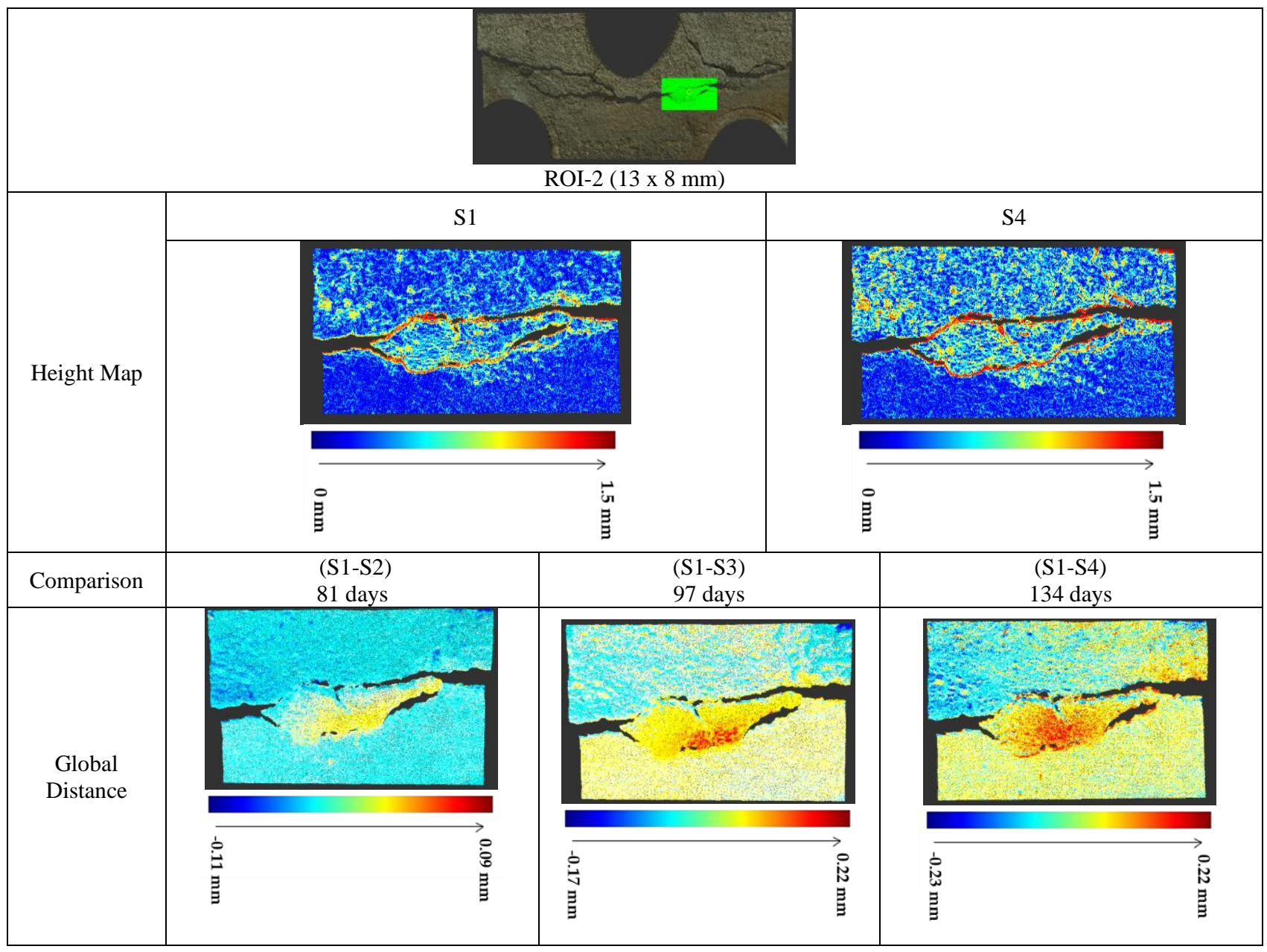




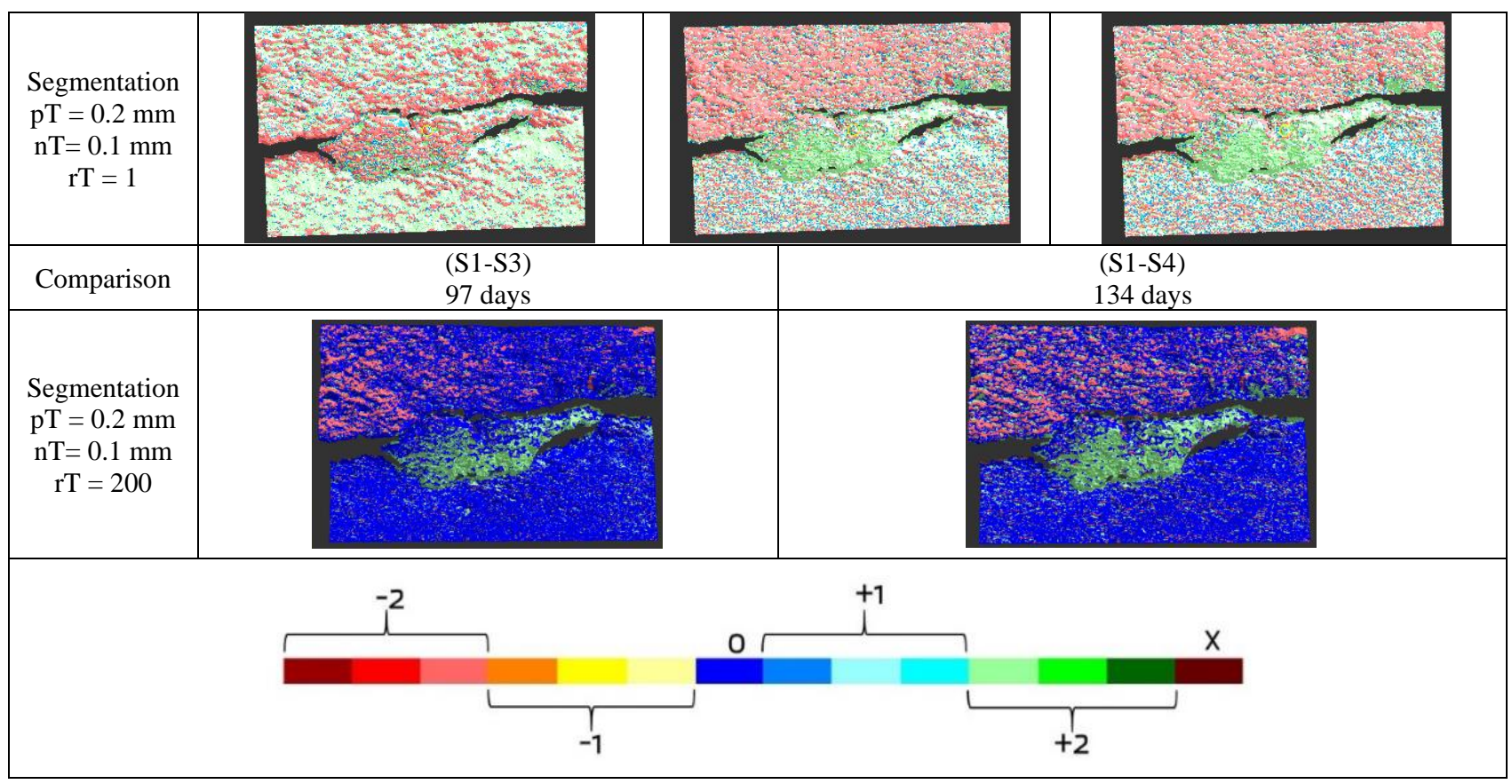

\section{Area No10}

As in the case of area No6, an area presenting visible surface damage from the reference state (S1) was monitored with the aim to detect and measure possible changes. From simple visual inspection of the 3D representation minor loss is detected after 134 days (Table 11).

Global distance calculations for the different time intervals (Table 12) show very small changes smaller than $0.5 \mathrm{~mm}$ mostly around the cracks and at the lower right part. The measured changes are of a very small scale to be able to withdraw results for the changes happening in the different time intervals. Regarding the comparison to the reference surface (Table 13), the main interest of observation is around the cracks where, between S1-S2 there appears to be a minor positive change that turns into maximum negative between S1-S3, whereas, in the last comparison S1-S4, areas of change are identified around the cracks with either positive or negative values. Also, in the S1-S4 material loss is identified in some areas (white, null).

Segmentation with default threshold (Table 14) is not presenting clear information on the changes of the surface. On the contrary segmentation with the user defined threshold (Table 15) shows the gradual degradation in the cracks that is resulting in loss of material and the small detachment. This process is presenting for the moment minor changes, but it characterises the trend of the surface change. The areas of change calculated in the global distance between S1-S4 can be considered too small $(0.3 \mathrm{~mm})$ to affect the segmentation.

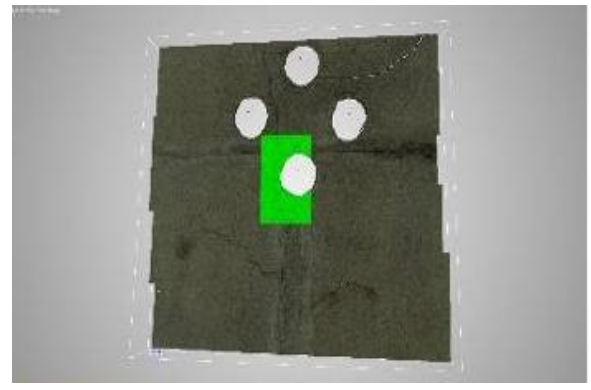

Figure5. Area No. 10C, Examined part dimension $28 \times 45 \mathrm{~mm}$. 
Table 11. Area No10- 3D mesh of the different time intervals

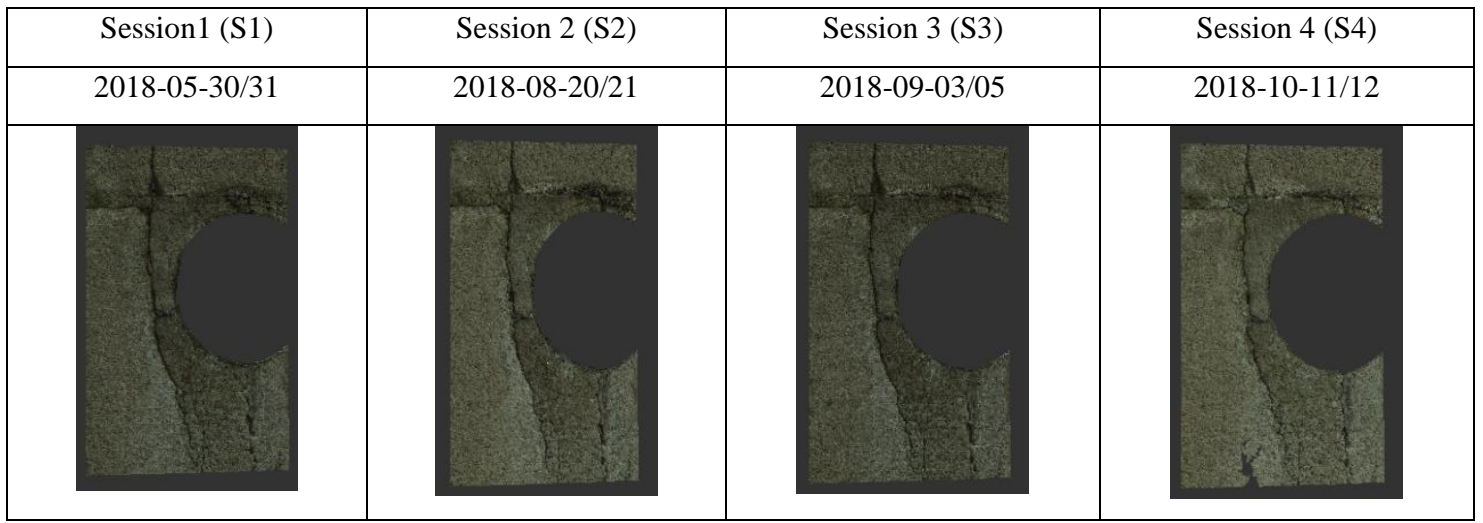

Table 12. Global Distance calculation between time intervals (default registration threshold)

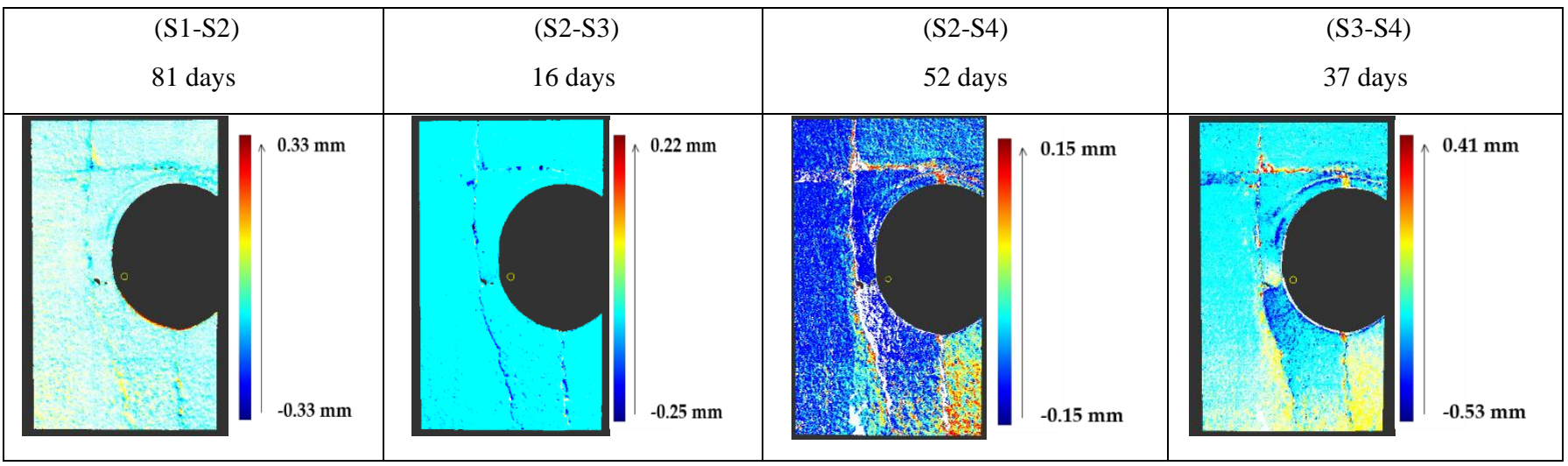

Table 13. Global Distance calculation from reference (default registration threshold)

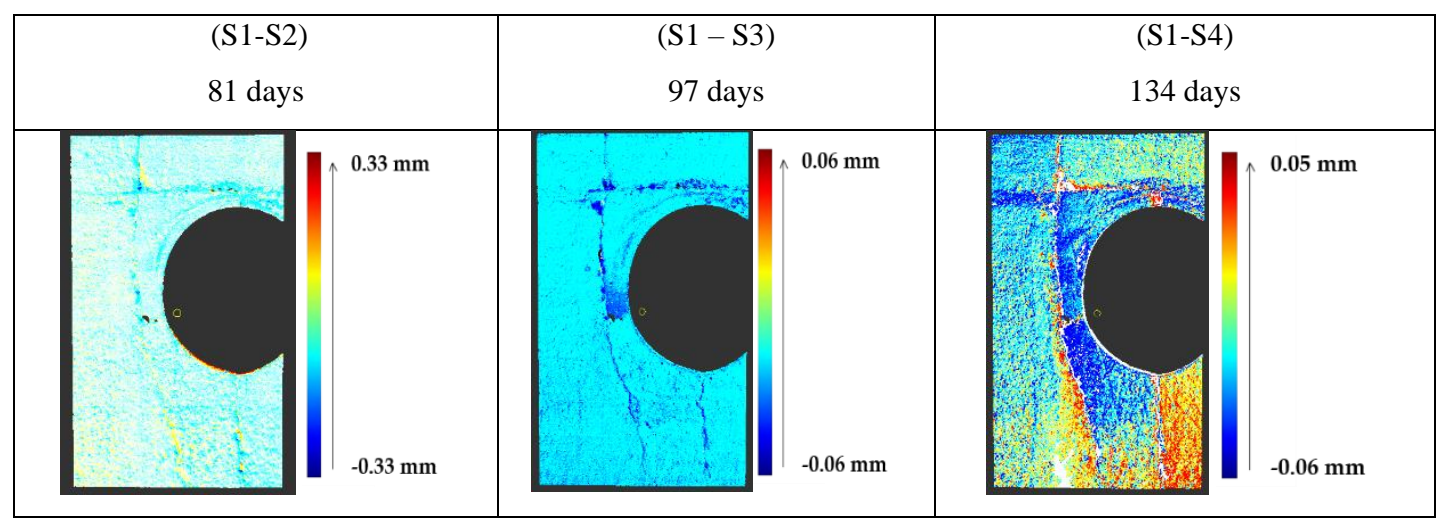


Table 14. Segmentation from reference value with default threshold $(\mathrm{pT}=0.5 \mathrm{~mm}, \mathrm{nT}=0.5 \mathrm{~mm}, \mathrm{rT}=1)$

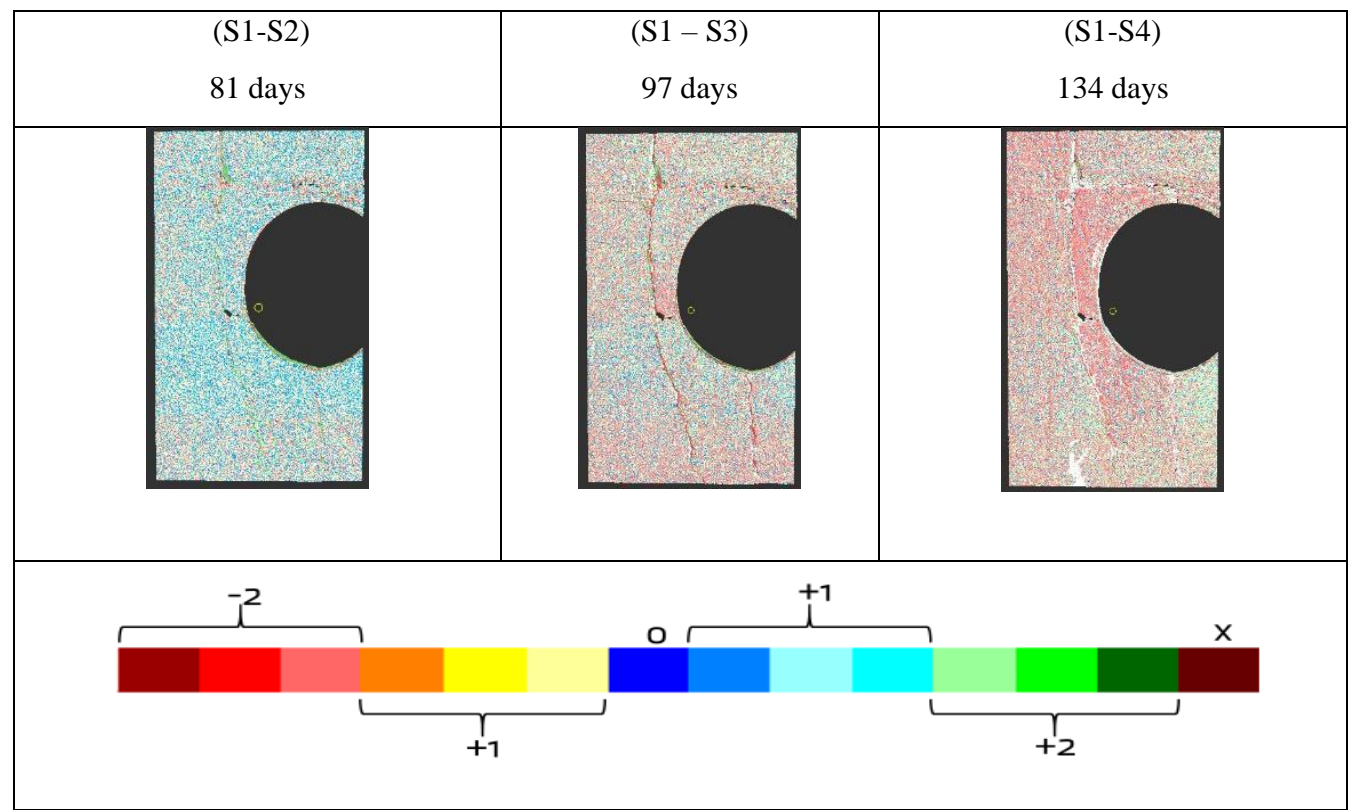

Table 15. Segmentation from reference value with user defined threshold $(\mathrm{pT}=0.2 \mathrm{~mm}, \mathrm{nT}=0.2 \mathrm{~mm}, \mathrm{rT}=200)$

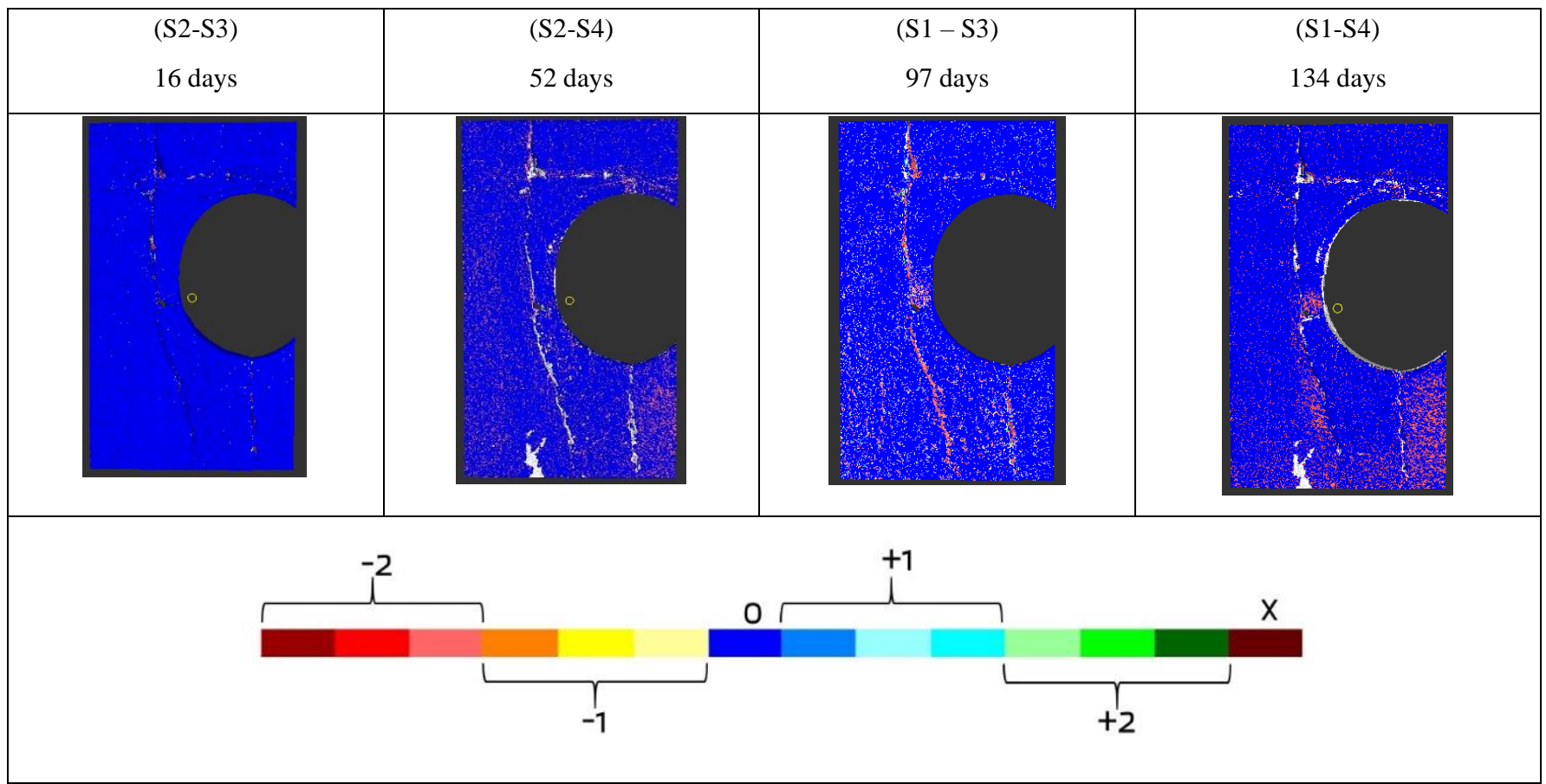

The examined methodology proposes a method of assessment of cross-time 3D point cloud data based on segmentation that presents high accuracy in measuring changes and clear visualizations of surface changes. Changes are measured based on height difference (z-axis) of the registered data corresponding mainly to oriented displacements of the reference surface in relation to degraded surface. For example, negative values could be assigned to material loss, whereas positive values 
could correspond to surface deposits. Equally, these displacements could be signs of movement of cracks in a particular direction corresponding to surface movement trends.

Appropriate registration for monitoring through 3D point clouds requires the use of markers since they can act as reference points. However, the use of markers in $\mathrm{CH}$ applications, especially permanent ones, is not desirable and is in some cases not applicable. For this reason, it is important to propose a methodology that can be applied on unregistered data. The main challenge in this case is to overcome the unstructured noisy large data and the requirement of mapping of each point of the points cloud in the same plane. The registration and fitting of the complex shaped data is a global problem in achieving accuracy in the segmentation results. The advantages of using segmentation for monitoring changes is that it is independent of the alignment errors and that the threshold can be modified based on the user's expertise or per case-study. Contrary to global distance calculation where the registration error cannot be avoided, in segmentation the alignment error can be excluded considering a reference ("no change") threshold and thus, avoid the cross-time registration error. Moreover, in global distance calculation the values are system defined and this needs to be considered when interpreting results since the scale is based on minimum and maximum values that may vary between time intervals and therefore assessing the visualized information, through the colour maps, may be misleading.

Therefore, when analysing datasets setting the appropriate rT threshold value is very crucial according to the examined surface and the anticipated change. Based on the experience of the material of the $\mathrm{CH}$ object and the monitoring periods, one can set the value of the registration threshold to obtain a better segmentation. However, this needs to be foreseen in any new acquisition. In unregistered data, setting such a value should be carefully considered and it is advisable, for the best practice of the application of the proposed methodology for monitoring, to set a reference $\mathrm{rT}$ value at the beginning of data acquisition.

For the presented case study, the areas of observation are considered too small to give a safe evaluation on the rate of degradation of the entire wall surface, however the applied methodology can record even small differences and present surface change trends. Regarding evaluating and analysing changes based on visual observation experts can detect significant changes corresponding to material loss but are unable to detect minor displacements in the order of a few micrometres.

Finally, the rT threshold was calculated based on approximation of the expected surface reaction to weathering, namely since measurable changes on a monumental surface within 16 days are very unlikely to happen, however this cannot be considered as ground truth for any dataset. In the Eq3 the registration threshold value was set, scaling to the ratio of N/knn and segmentation was performed assuming that the quality of the cross-time registration is the same for each dataset.

\section{CONCLUSION AND FUTURE PERSPECTIVE}

A computational approach for change detection of surface geometry of $\mathrm{CH}$ surfaces and an automated grouping based on the similarity of surface geometry change is presented in this work through the proposed segmentation methodology. This methodology is developed for analysing and detecting changes on the surface of $\mathrm{CH}$ objects with high resolution of surface details based on surface points in 3D space and therefore resulting in high accuracy measurements that can quantify information that are not easily detectable through visual observation. For a holistic evaluation of the surface condition a combination of different types of data and expertise is required to draw safe conclusions.

Comparisons between height maps, global distance visualisations and the proposed segmentation methodology are demonstrating the visualisation and quantification abilities of segmentation. Especially, through the possibility to define the appropriate thresholds that can help in an even better visualisation and understanding of the surface changes.

Segmenting geometric behaviour of $\mathrm{CH}$ surfaces over time is a fruitful way of analysing and representing various types of changes during the monitoring period and demonstrate different behaviours of changes for each phase, like material loss, accumulations of surface deposits or general movement trends. Furthermore, the computational analysis of each surface point and the comprehension of the respective neighbouring points' behaviour can give the estimation of local geometry change comparing more than one phase of data and therefore lead to better understanding the change trends over time for the examined surface. 


\section{ACKNOWLEDGEMENT}

This work is carried out by the beneficiary partner of CHANGE-ITN (Cultural Heritage Analysis for New GenerationInnovative Training Network) at Warsaw University of Technology, Poland, supported and funded by the European Union's Horizon 2020 research and innovation program under the Marie Skłodowska-Curie grant agreement No. 813789. The financial support through the project granted by the Scientific Council of the Discipline Mechanical Engineering, WUT is greatly appreciated. The authors would also like to acknowledge the people working at the Museum of King Jan III's Palace at Wilanów, Warsaw Poland for their collaboration and for providing access to the data from the Project entitled "Restoration and protection of the symbol of the Polish cultural heritage - the Museum of King Jan III's Palace in Wilanów", co-financed by the European Union from the European Regional Development Fund under the Operational Program Infrastructure and Environment.

\section{REFERENCES}

[1] ICOMOS, "Charter of the Principles for the recording of monuments, groups of building and sites", 1996, $\langle$ https://www.icomos.org/charters/archives-e.pdf > (May 2021)

[2] Sellem L. (ed.), [Monitoring World Heritage], UNESCO- World Heritage Center, (2004) <https://whc.unesco.org/en/documents/4618> (May 2021)

[3] Letellier R., Schmid W., Le Blanck F., [Recording, Documentation and Information Management for the conservation of heritage places], Getty Conservation Institute, ISBN 978-0-89236-925-6, (2007) <http://www.getty.edu/conservation/publications_resources/pdf_publications/pdf/guiding_principles.pdf >.

[4] CEN/TC 346- EN 15898:2019, Conservation of Cultural Heritage-Main general terms and definitions.

[5] Sitnik R., Lech K., Bunsch E., and Michoński J., "Monitoring surface degradation process by 3D structured light scanning", Proc. SPIE 11058, Optics for Arts, Architecture, and Archaeology VII, 1105811 (12 July 2019); https://doi.org/10.1117/12.2525668.

[6] Tobiasz, A.; Markiewicz, J.; Łapiński, S.; Nikel, J.; Kot, P.; Muradov, M., "Review of Methods for Documentation, Management, and Sustainability of Cultural Heritage. Case Study: Museum of King Jan III's Palace at Wilanów. Sustainability", 11, 7046 (2019). https://doi.org/10.3390/su11247046

[7] Grilli, E.; Remondino, F., "Classification of 3D Digital Heritage", Remote Sens. 11, 847 (2019). https://doi.org/10.3390/rs11070847.

[8] Girardeau-Montaut D., Roux M., Raphael M., and Thibault G., "Change Detection on points cloud data acquired with a ground laser scanner", ISPRS WG III/3, III/4, V/3 Workshop "Laser scanning 2005", 30-35 (2005).

[9] Lague D., Brodu N., Leroux J., "Accurate 3D comparison of complex topography with terrestrial laser scanner: Application to the Rangitikei canyon (N-Z)", ISPRS P\&RS, 82, 10-26 (2013), ISSN 0924-2716, https://doi.org/10.1016/j.isprsjprs.2013.04.009.

[10] Lercari N, "Monitoring earthen archaeological heritage using multi-temporal terrestrial laser scanning and surface change detection, Journal of Cultural Heritage", 39, 152-165 (2019), ISSN 1296-2074.

[11] Bruno F., Gallo A., De Filippo F., Muzzupappa M., B. Davidde Petriaggi and P. Caputo, "3D documentation and monitoring of the experimental cleaning operations in the underwater archaeological site of Baia (Italy)", Digital Heritage International Congress (Digital Heritage), Marseille, 105-112 (2013).

[12] F. Peteler, E. Gattet, P. Bromblet, O. Guillon, J. Vallet and L. De Luca, "Analyzing the evolution of deterioration patterns: A first step of an image-based approach for comparing multitemporal data sets", Digital Heritage, Granada, 113-116 (2015).

[13] Abate D., "Built-Heritage Multi-temporal Monitoring through Photogrammetry and 2D/3D Change Detection Algorithms, Studies in Conservation", 64(7), 423-434 (2019), DOI: 10.1080/00393630.2018.1554934.

[14] Murtiyoso, A. and Grussenmeyer, P., "Automatic heritage building point cloud segmentation and classification using geometrical rules”, Int. Arch. Photogramm. Remote Sens. Spatial Inf. Sci., XLII-2/W15, 821-827 (2019), https://doi.org/10.5194/isprs-archives-XLII-2-W15-821-2019.

[15] Bassier, M., Bonduel, M., Van Genechten, B., and Vergauwen, M., "Segmentation of large unstructured point clouds using octree-based region growing and conditional random fields", Int. Arch. Photogramm. Remote Sens. Spatial Inf. Sci., XLII-2/W8, 25-30 (2017), https://doi.org/10.5194/isprs-archives-XLII-2-W8-25-2017.

[16] Su, Y. T., Bethel, J. and Hu, S., "Octree-based segmentation for terrestrial LiDAR point cloud data in industrial applications", ISPRS P\&RS, 113, 59-74 (2016). 
[17] Liu H., Song R., Zhang X., and Liu H., "Point clouds segmentation based on Euclidean clustering and multi-plane extraction in rugged field", Meas. Sci. Technol., (2021), http://iopscience.iop.org/article/10.1088/1361-6501/abead3.

[18] Di Angelo L.,and Di Stefano P.. "Geometric segmentation of 3D scanned surfaces.” Comput. Aided Des., 62, 44-56 (2015).

[19] Pilario, K.E.; Shafiee, M.; Cao, Y.; Lao, L.; Yang, S.-H, “A Review of Kernel Methods for Feature Extraction in Nonlinear Process Monitoring”, Processes 2020, 8, 24. https://doi.org/10.3390/pr8010024.

[20] Wang X., Xing E., and Schaid D., "Kernel methods for large-scale genomic data analysis", Briefings in Bioinformatics, 16(20), 183-192 (2015), https://doi.org/10.1093/bib/bbu024.

[21] https://www.wilanow-palac.pl/palace.html (May 2021). 